\title{
Performance of Corrected Imaging Energy Filters
}

\author{
Erik Essers, Dieter Krahl, Bernd Huber, Alexander Orchowski
}

LEO Elektronenmikroskopie GmbH, D-73446 Oberkochen, Germany

We present experimental results concerning the adjustment strategy of two new types of corrected imaging energy filters as incorporated in the new generation of LEO's Energy Filtering Transmission Electron Microscopes (EFTEM).

A second-order corrected and in third order minimized $90^{\circ}$ OMEGA type energy filter has been operating for one year in a 200kV LEO EFTEM with a FEG [1]. The main properties of the filter are a dispersion of $1.85 \mu \mathrm{m} / \mathrm{eV}$ at $200 \mathrm{kV}$ and a high acceptance, which is described by the transmissivity $\mathrm{T}_{\text {real }}$ [2]. Due to the aberration correction, this "in column" filter provides the high transmissivity of $\mathrm{T}_{\text {real }}=320 \mathrm{~nm}^{2} / \mathrm{eV}$ at $200 \mathrm{kV}$ at an energy window width of $1 \mathrm{eV}$. This allows one to transfer large and highly resolved energy filtered image fields with very good isochromaticity.

Within the SESAMe (Sub-eV-Sub-Ångstrom Microscope) project [3] LEO developed the Mandoline energy filter, which was proposed by Uhlemann and Rose [4]. The energy resolution of $0.2 \mathrm{eV}$ requires a filter current stability of $2 * 10^{-7}$. The same energy spread is required for the primary electron beam. The SESAMe EFTEM therefore provides a high tension stability of $5^{*} 10^{-7}$ and incorporates a FEG with an integrated monochromator [5] which allows for a primary beam energy width of less than $0.2 \mathrm{eV}$.

The Mandoline energy filter sets a new standard for high end energy filtering TEM. This third-order corrected "in column" energy filter provides the high dispersion of $\mathrm{D}=6.2 \mu \mathrm{m} / \mathrm{eV}$ at $200 \mathrm{kV}$ and the unrivalled transmissivity of $T_{\text {real }}=3300 \mathrm{~nm}^{2} / \mathrm{eV}$ at an energy window width of $1 \mathrm{eV}$. An extremely good isochromaticity even for very large image fields is thus achieved.

The Mandoline filter (figure 1) consists of a homogeneous magnet M1, two symmetrically arranged inhomogeneous magnets M2, M3 and nine symmetrically arranged correction elements C1, ... C9. It combines a short length with the excellent imaging properties of conical deflection magnets (M2, M3) focussing the ray continuously in both principal sections. This leads to a smoother path of the fundamental rays thus providing smaller aberrations. Despite its very high transmissivity and its large deflection radius $(75 \mathrm{~mm})$, the total length of the Mandoline filter $(228 \mathrm{~mm}$ between the diffraction plane and the energy selection plane) is comparable to that of conventional imaging energy filters. A high mechanical stability of the TEM column is therefore attainable. The short total length is due to the path of the optical axis which intersects itself within the homogeneous magnet M1 of the filter.

The aberrations of the filter are eliminated by multipole fields at the correction elements. Half of the second-order aberrations vanish due to the filter's symmetry. The remaining second-order aberrations in the achromatic image plane and energy selection plane are eliminated by symmetrically arranged hexapole fields at the nine correction elements. Apart from C3 and C7, the correction elements are positioned at the zeros of the fundamental rays. This decouples the effect of the correction elements on the second-order aberrations to a large extent, thus simplifying the correction procedure. Simultaneously with the second-order correction of the geometric aberrations 
the most disturbing chromatic aberrations at the achromatic image plane vanish and additionally the third-order distortions and the third-order comas vanish due to the filter's symmetry [6]. The correction of the third-order axial aberrations at the energy selection plane is performed by octopole fields at selected correction elements. The order of the aberration correction steps is such, that a corrected aberration in general is not influenced by the subsequent correction steps. Only for selected steps does a weak coupling exist, resulting in a repetition of this part of the aberration correction procedure. The criteria to judge the correction state in each step and the sequence of the correction steps lead to a well-defined overall alignment strategy for the Mandoline energy filter.

We present the properties of the Mandoline energy filter and aspects of the alignment and the correction procedure. Experimental examples at $200 \mathrm{kV}$ are provided for demonstration.

[1] G. Lang, G. Benner (2002) Microsc. Microanal., this volume.

[2] S. Uhlemann, H. Rose (1996) Ultramicroscopy 63, 161

[3] M. Rühle et al. (2000) Microsc. Microanal. 6

[4] S. Uhlemann, H. Rose (1994) Optik 96, 163

[5] FEG and monochromator are custom developed by CEOS for LEO;

S. Uhlemann, M. Haider(2002) Microsc. Microanal., this volume.

[6] H. Rose, D. Krahl (1994) Springer Series in Optical Sciences 75, 42

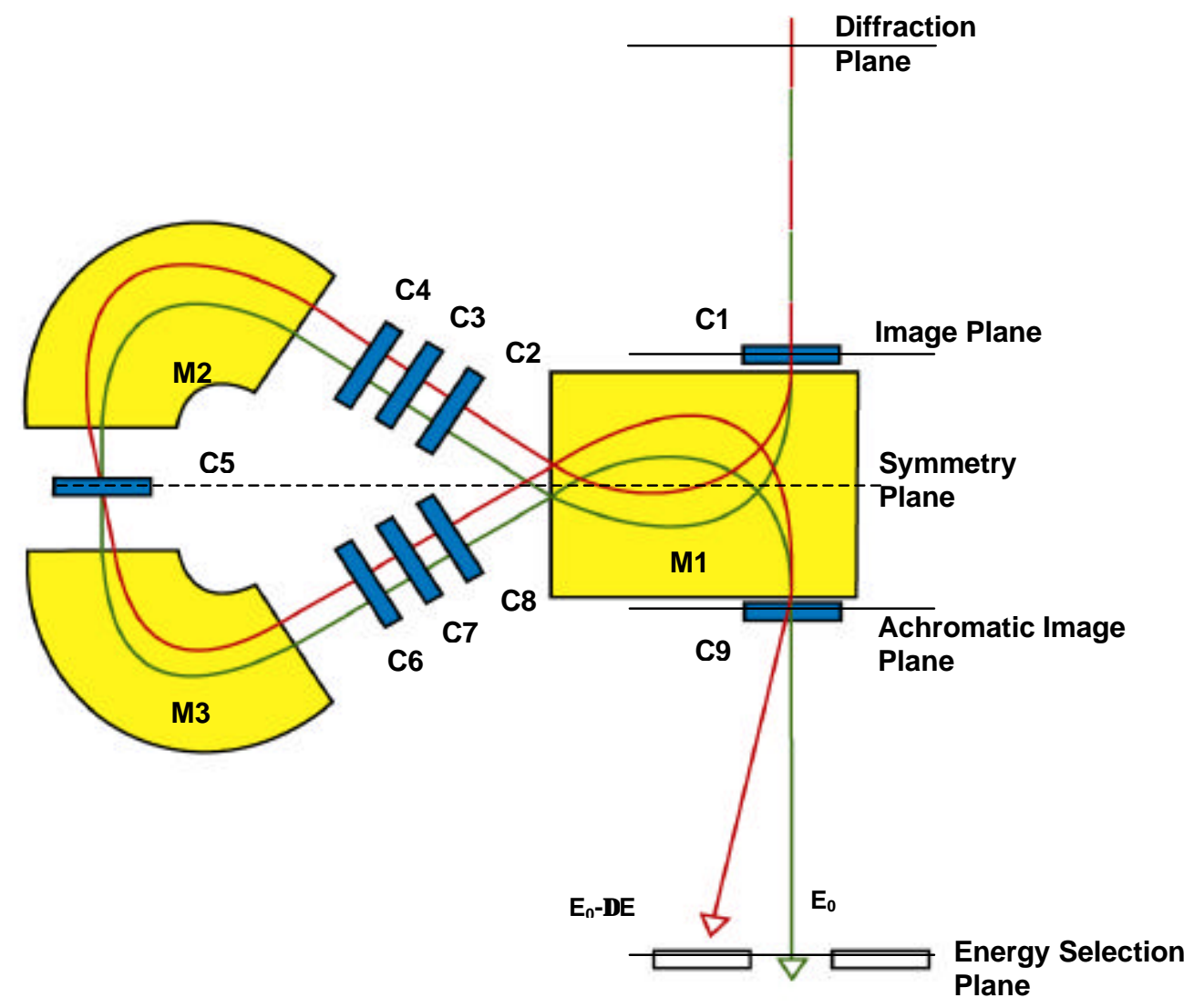

Figure 1: Main components of the Mandoline energy filter (schematic diagram)

Corresponding author: Essers@leo.de 